\title{
Patterns of Woody Species Diversity Along Disturbance Gradient in Gumdri Abo Natural Forest, Dangila District, North Western Ethiopia
}

\author{
Getnet Bitew $^{1^{*}} \quad$ Amare Tesfaye ${ }^{2}$ \\ 1.Department of Natural Resource Management, Debark University College of Agriculture and Environmental \\ Science (DKU CAES), P.O Box 90, Debark, North Gondar, Ethiopia \\ 2.Department of forestry, Hawassa University Wondo Genet College of Forestry and Natural Resources \\ (WGCF-NR), P.O.Box 128, Shashemene, Ethiopia
}

\begin{abstract}
The study was conducted in Gumdri Abo natural forest along the three disturbance gradients which is located in Dangila Woreda Amhara Region Ethiopia. The aim of the study was to determine woody species diversity and stand structure within the three forest strata that were classified based on human induced forest disturbance level. For vegetation analysis, the forest was stratified in to three disturbance classes, i.e. Heavily Disturbed Forest (HDF), Moderately Disturbed Forest (MDF) and Low Disturbed Forest (LDF). Data were collected from nested circular plots of $11.28 \mathrm{~m}\left(400 \mathrm{~m}^{2}\right)$ for trees and $5.64 \mathrm{~m}\left(100 \mathrm{~m}^{2}\right)$ plots for seedlings and saplings that are laid down systematically along six transect lines. Equal sample plots were allocated for each forest strata. Woody species diversity was analyzed using Shannon Diversity Index (H'). Woody species density and diversity were assessed for each disturbance classes. One-way ANOVA was used to test significances differences among disturbance classes. A total of 72 woody species belonging to 40 families and 62 genera were recorded. Trees, shrubs and lianas account $44.4 \%, 45.8 \%$ and $9.7 \%$ respectively. Richness and diversity of woody species decreased from LDF to HDF. The diversity and density of woody species in the three forest strata were significantly different at $95 \%$ confidence interval. Stand structure from diameter class distribution for trees and shrubs revealed trends of normal $\mathrm{J}$ shape in the three forest strata. The findings of the study indicated that woody species diversity and density in HDF and MDF were significantly lower than LDF. Thus, collaborative form of forest conservation by the government aimed at woody species conservation should be geared towards protecting Gumdri Abo natural forest from excessive human disturbance.
\end{abstract}

Keywords: Conservation, Disturbance, Gradient, Diversity, woody species, Density

DOI: $10.7176 / \mathrm{JNSR} / 9-3-05$

\section{INTRODUCTION}

Loss of forest cover and biodiversity due to anthropogenic activities is a growing concern in many parts of the world (Hegde \& Enters, 2000). Africa's forest cover is estimated to be 650 million ha, constituting $17 \%$ of the world's forests including a number of international biodiversity hotspots. More than $50 \%$ of African land is covered by Afromontane vegetation, which is a type of vegetation found on highlands of Africa (Coetzee, 1978).

In Africa mountain forests are important to protect rare species because they contain unique ecosystems with exceptional species richness and high levels of endemism. Knowing species richness patterns is crucial to provide insights to environmental planners, nature reserve designers, ecologists and botanists. The structure of plant community in many natural ecosystems is largely determined by the disturbances, which occur quite frequently. An understanding of species distribution patterns and the resulting spatial patterns of biodiversity is fundamental to the study of not only evolutionary biology but also of conservation biology (Armesto \& Pickett, 1985; Sang, 2009).

Ethiopia is regarded as one of the most important countries in Africa with respect to floral and faunal biological resources (EFAP, 1994). The number of species of higher plants found in Ethiopia is about 6000, of which about $10 \%$ are endemic to the country (Vivero et al., 2006). The occurrence of isolated mature trees in farmlands and the patches of forests that are seen around church yards and religious burial grounds indicate the presence of vast expanse of forests earlier in Ethiopia. This is because of the church yards play a role in forest conservation practice in Ethiopia (Tamrat, 1993).

The country has a forest cover of about 12.4milion hectares (11.5\%). Between 1990 and 2000, the extent of Ethiopia's forests (both forests and woodland) decreased by 1.4 million hectares. By 2005, the forest cover had further declined and was estimated to cover 13.0 million hectares. In other words, Ethiopia lost over two million hectares of forest per year with an average loss of 140, 000 hectares (FAO, 2015).

Therefore, the country had experienced substantial deforestation, soil degradation and an increase in the area of bare land over the years. The need for fuel wood, arable land and grazing areas have been indicated as the main causes of forest disturbance and leading to loss of forest biodiversity related constraints (Ensermu \& Teshome, 2008). Disturbances created by these activities influence forest dynamics and tree density at the local and regional 
scales. In the face of these problems, conservation biologists have sought to protect forests using several different strategies from strict protection in National Parks, to sustainable forest management and other integrated conservation and involving traditional conservation methods and development program (Ramire et al., 2001).

The structure and function of forest ecosystem is determined by the plant component more than any other living component of the system (Richards, 1996). The plant diversity at any site is influenced by species distribution and abundance patterns (Palit \& Chanda, 2012). And the richness of plant species is controlled by a variety of biotic and abiotic parameters (Huston, 1994). Topography, soil, climate, anthropogenic disturbance and geographical location of a region influence the vegetation diversity of the forest ecosystem (Ram et al., 2004).

Lack of integration of the local people living around the conservation areas into the conservation efforts is the major constraint to the overall conservation effort in Ethiopia (Feyera \& Demel, 2003). Because of this, it has now been realized that unless the local community is involved in the conservation effort, the sustainability of forest resource will be under question.

Gumdri Abo natural forest is found in Dangila district, North Western Ethiopia. The forest has a characteristics of species found in dry Aforomontane vegetation type of Ethiopia. Common woody species in this forest includes Juniperus procera, Prunus africana, Euphorbia spp. Carissa spinarum, Rosa abyssinica, and Acacia abyssinica. These species are also common and an indication of dry Afromontane vegetation type in Ethiopia (Sebsebe \& Friis, 2009).

Gumdri Abo natural forest comprises of Gumdri Abo Orthodox church forest at the middle, which is relatively well conserved. However, illegal logging, fuel wood collection and overgrazing poses a serious threat to the survival of the forest (forest outside the church). As a result, most of the forest area is degraded currently by anthropogenic disturbances as its root cause. Unless conservation measure is taken, the forest coverage will shrink and converted to other land use types by the near future. Therefore, the present study aimed at generating information on patterns of woody species diversity, richness and stand structure along disturbance gradients in Gumdri Abo natural forest.

\section{METHODS}

\subsection{Study area}

The study was carried out in Gumdri Abo natural forest in Dangila woreda, Northwestern Ethiopia. Geographically, the study area is found between $11^{\circ} 9^{\prime}-11^{\circ} 15^{\prime} \mathrm{N}$ to $36^{\circ} 45^{\prime}-36^{\circ} 57^{\prime} \mathrm{E}$. Dangila district is found $78 \mathrm{~km}$ south west from Bahir Dar and $485 \mathrm{~km}$ from Addis Ababa. It is bordered on the south by Faggeta Lekoma, on the Southwest by Guangua, on the Northwest by Jawi, and on the Northeast by Mirab Gojjam Zone and it is located with an altitude of $2200 \mathrm{~m}$ a s 1 (DWAO, 2014). 


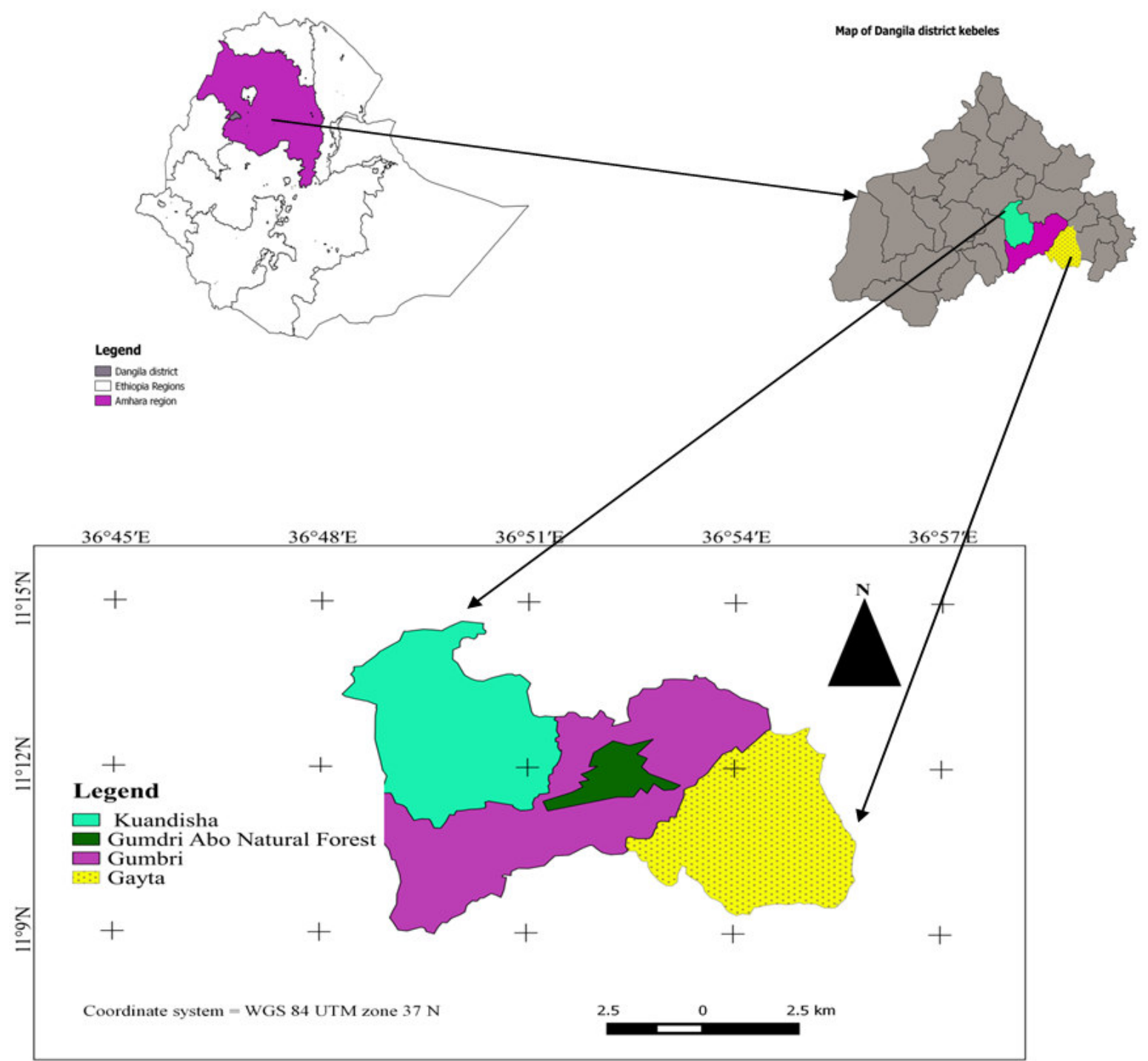

Figure 1: Location map of the study area

The agro ecology of the forest is characterized by moist Weina-Dega (mid-land). The area receives a mean annual rainfall of $1578 \mathrm{~mm}$. The district experiences a mean annual temperature of $17{ }^{\circ} \mathrm{C}(\mathrm{DWAO}, 2014)$.

Croton macrostachyus, Acacia lahai, Eucalyptus camaldulensis, Cordia africana, Albizia gummifera, Sesbania sesban and Justicia schimperiana form the dominant vegetation types in the area. Most of those vegetations are majorly confined in Churches. Gumdri Abo natural forest covers the area about 62.25 ha. Some species like; Juniperus procera, Prunus africana, Euphorbia spp. Carissa spinarum, Rosa abyssinica, and Acacia abyssinica cover most part of the forest. Such species are most common and a characteristic feature of dry Afromontane vegetation type in Ethiopia (Sebsebe \& Friis, 2009).

\subsection{Sampling}

A reconnaissance survey was carried out to obtain an impression of the site conditions and to determine the sampling methods for vegetation inventory. During this time overall information on the study site was obtained and sampling method to be used was determined. As a result, systematic sampling method was used to collect vegetation data. This technique was employed to ensure representative samples from all forest types.

The study was carried out in three forest types that were selected based on the levels of human disturbance along that gradient. These were the LDF, MDF and HDF. The disturbance gradients were selected to show the relative patterns of woody species diversity in the three forest types. The area coverage of each forest type is different that consists of 12, 19.25 and 31 hectares for LDF, MDF and HDF respectively.

The LDF is owned by Gumdri Abo Orthodox church. This forest is categorized as a primary forest because it is part of the forest that has not undergone any major form of human disturbance. This is partly due to the fact that this forest type is part of church forest (Gumdri Abo Orthodox Church) in better protection from disturbance. 
The MDF and HDF are the degraded parts of the original/primary forest that has undergone various levels of human disturbances by the local community. The MDF is relatively less disturbed with human disturbances in the form of illegal logging and activities that had taken place. On the other hand, recent disturbances in the form of logging, fuel wood collection and overgrazing have characterized the HDF.

Six transect lines were laid on the three forest types (two for each) for vegetation inventory. Circular sample plot size of $11.28 \mathrm{~m}$ radius approximately $400 \mathrm{~m}^{2}$ was established systematically at every $50 \mathrm{~m}$ interval along the transect lines. The center of each plot was marked using a wooden peg and plot radius was measured using measuring tape. Woody plant species found outside sample plots within $10 \mathrm{~m}$ distance were recorded for the purpose of complete plant species documentation in the study area (Mueller \& Ellenberge, 1974; Kent \& Coker, 1992).

To assess woody species diversity and composition in the forest, stratified systematic sampling procedure was used. Accordingly, the forest was stratified in to three forest strata based on site conditions as HDF, MDF and LDF. These forest strata are more or less similar in topography and agro ecology. Disturbance intensities were also calculated as the ratio of the number of cut stumps to total number of individual trees for site classification criteria (Addo-Fordjour et al., 2009). Accordingly, the disturbance intensities calculated were 0.02, 0.43 and 0.95 for LDF, MDF and HDF respectively. A total of 30 circular sample plots (10 from each forest strata) were used for vegetation inventory. Equal sample plot allocation technique in each forest strata (10 plots each) was applied for comparison purpose. The initial sample plot was taken randomly in $10 \mathrm{~m}$ distance away from the edge of forest to minimize boundary effect.

All woody species encountered in each sample plot were recorded in their local and scientific names. Diameter measurement for trees and lianas was taken at breast height $(1.3 \mathrm{~m})$ using Caliper. Trees that were branched at the breast height, diameter was measured separately above the swelling and averaged. For tree species forked below $1.3 \mathrm{~m}$, individual stems were separately measured and treated as two trees (Abed and Stephens, 2003). In each plot, trees with $\mathrm{DBH} \geq 10 \mathrm{~cm}$ were measured and recorded (Plate 1).

Shrub diameter measurement was taken at stump height with DSH $\geq 2.5 \mathrm{~cm}$ (DSH) at $30 \mathrm{~cm}$ ground level (Mac, 1997). In the case of multi-stemmed shrub each stem was measured and diameter equivalent of the plant calculated as the square root of the sum of diameter of all stems per plant (Snowdon et al., 2002), $d=\sum_{i}^{n} \sqrt{\mathrm{di}^{2}}$ where: $d=$ diameter equivalent height, $d i=$ diameter of the $i^{\text {th }}$ stem at the measurement height $(\mathrm{cm})$. The height of trees, shrubs and lianas were measured using hypsometer.

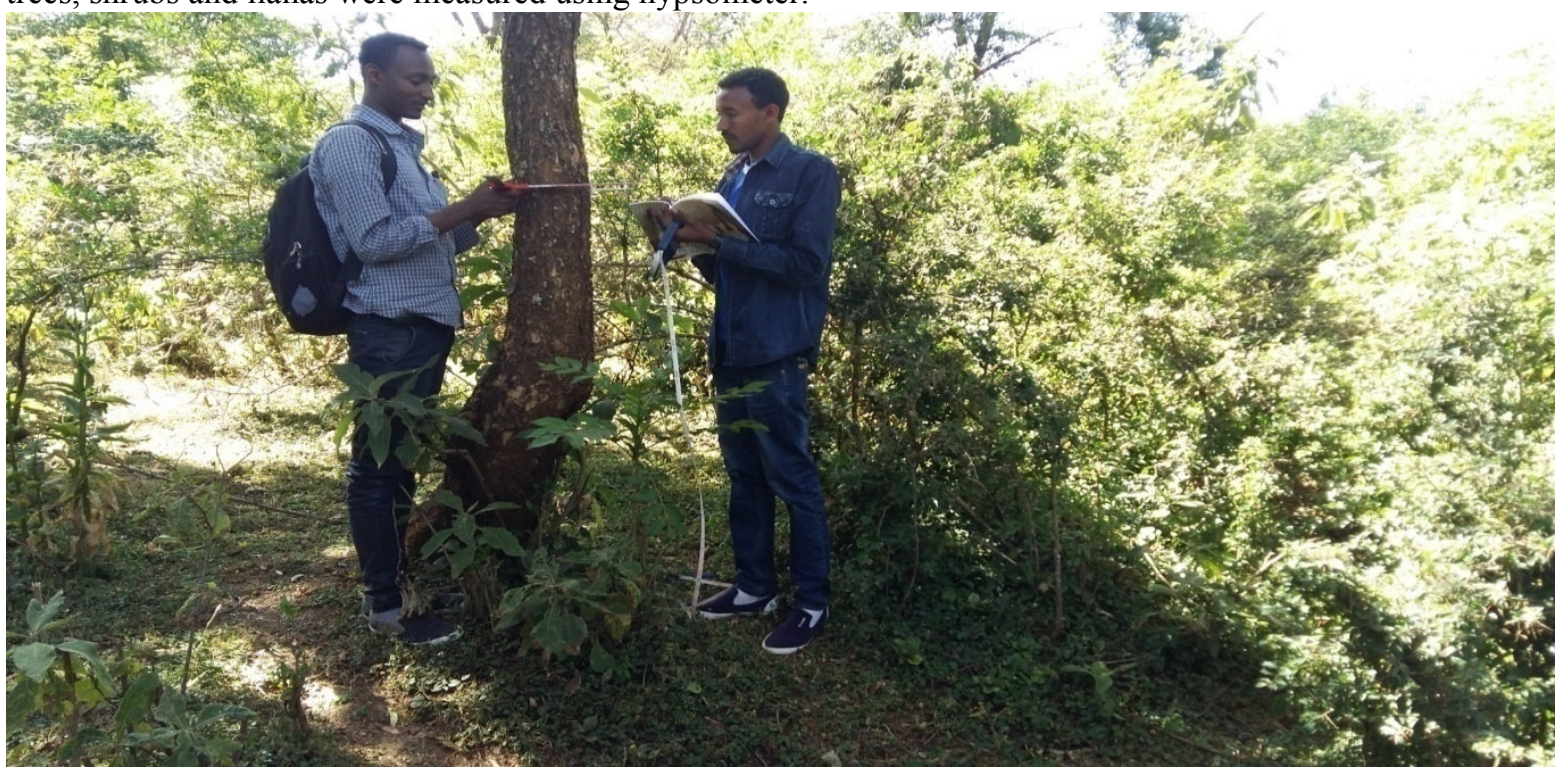

Plate 1: DBH measurement in the field during data collection

Scientific nomenclature was carried out using published volumes "Flora of Ethiopia and Eritrea" (Sebsebe, 1997), Useful Trees and Shrubs of Ethiopia (Azene, 2007) and Natural Database for Africa (NDA) Version 2 (Ermias \& Emeritu, 2011). For some species that were unable to identify directly in the field plant specimens were collected, pressed, dried and brought to National Herbarium of Ethiopia, Department of Plant Biology and Biodiversity Management, Addis Ababa University for further identification.

\subsection{Data Analysis}

One-way ANOVA was used to test the differences in woody species diversity and density between the three forest types. In addition to this, descriptive statistics was also used to show proportion of stand parameters. The analysis 
was performed using Statistical package for Social Science (SPSS) Version 20.0 software at a significance level of 5\%. Tables and Figures were used to present the result of this study.

Species richness is the total number of different woody species recorded in each of the sampled plots (Magurran, 1988). Accordingly, in this study species richness was determined by the total number of woody species recorded in the three forest types. The diversity of woody species was analyzed by using Shannon Weiner diversity index $\left(\mathrm{H}^{\prime}\right)$.

$\mathrm{H}^{\prime}=-\sum_{i=1}^{S}(\mathrm{Pi} \ln \mathrm{Pi})$

Where; $\mathrm{H}^{\prime}=$ Shannon species diversity Index, $\mathrm{S}=$ number of species, $\mathrm{Pi}=\mathrm{n} / \mathrm{N}$ is the proportion of individuals found in the $\mathrm{i}^{\text {th }}$ species (ranges 0 to 1 ), $\mathrm{n}=$ number of individuals of a given species, $\mathrm{N}=$ total number of individuals found and $\mathrm{Pi}=$ proportion of $\mathrm{S}$ made up of the $\mathrm{i}^{\text {th }}$ species (Shannon and Wiener, 1949)

Shannon evenness $(\mathrm{J})=\frac{\mathrm{H} \prime}{\mathrm{H} \prime \max }$

Where; $\mathbf{J}=$ Evenness, $\mathrm{H}^{\prime}=$ Shannon-Wiener diversity index and H'max $=\ln \mathrm{S}$ where $\mathrm{S}$ is the number of species.

The structure of the vegetation was described based on the analysis of species density, DBH, height, basal area, frequency and important value Index (IVI). Diameter distribution of trees and shrubs in the three forest types was classified into nine diameter classes. For the description of diameter distribution, individual trees with DBH $\geq 10 \mathrm{~cm}$ and shrubs $\geq 2.5 \mathrm{~cm}$ were selected as Samson et al., (2010).

Density (D) of woody plant species was calculated by using the following formula;

$$
\begin{aligned}
& \qquad \mathrm{D}=\frac{\text { Number of indiviguals woody species }}{\text { Sum of all plot areas }} \\
& \text { Relative density }(\%)=\frac{\text { Total number of individuals of species A }}{\text { Total number of individuals of all species }} \times 100
\end{aligned}
$$

Frequency (F) of plant species was calculated by using the following formula;

$$
\begin{gathered}
\mathrm{F}=\frac{\text { Number of quadrats in which a species occur }}{\text { Total number of quadrats sampled in the study site }} \times 100 \\
\text { Relative frequency }(\%)=\frac{\text { Frequency of species } \mathrm{A}}{\text { Frequency of all species }} \times 100
\end{gathered}
$$

Basal area (BA): It is the area outline of a plant near ground surface for trees. The analysis of tree basal area was made using basal area measurements.

$$
\begin{gathered}
\mathrm{BA}=\frac{\pi d^{2}}{4} \text { Where; } \mathrm{BA}=\text { Basal Area in } \mathrm{m}^{2} \text { per hectare, } \mathrm{d}=\text { diameter at breast height in meter and } \pi=3.14 \\
\text { Relative dominance }(\%)=\frac{\text { Basal area of species } \mathrm{A}}{\text { Total basal area of all species }} \times 100
\end{gathered}
$$

IVI of woody species was calculated from the sum RDO, RD and RF (Kent and Coker, 1992). Mathematically it can be expressed as follows;

$$
\mathrm{IVI}=\mathrm{RBA}+\mathrm{RD}+\mathrm{RF}
$$

\section{RESULT}

\subsection{Woody Species Composition}

A total of 72 woody species representing 40 families and 62 genera were recorded in Gumdri Abo natural forest. Out of these, 67 woody species were recorded inside sampled plots and 5 plant species were recorded outside the sampled plot. From the total recorded species 22, 40, and 56 species were recorded from HDF, MDF and LDF respectively. The collected woody species were composed of trees and shrubs accounting $44.4 \%$ and $45.8 \%$ respectively in the entire forest. Out of the total species composition, trees share $36.4 \%, 42.5 \%$ and $48.2 \%$ in the HDF, MDF and LDF respectively. Similarly, Shrubs share $54.5 \%, 45 \%$ and $41.1 \%$ in the HDF, MDF and LDF respectively (Table 1). 
Table 1: Summary of woody species composition in Gumdri Abo natural forest

\begin{tabular}{|c|c|c|c|c|c|c|c|c|}
\hline \multirow[b]{2}{*}{ Growth habit } & \multicolumn{2}{|l|}{ HDF } & \multicolumn{2}{|l|}{ MDF } & \multicolumn{2}{|l|}{ LDF } & \multicolumn{2}{|c|}{ Over all } \\
\hline & Number & $\%$ & Number & $\%$ & Number & $\%$ & Number & $\%$ \\
\hline Tree & 8 & 36.4 & 17 & 42.5 & 27 & 48.2 & 32 & 44.4 \\
\hline Shrub & 12 & 54.5 & 18 & 45.0 & 23 & 41.1 & 33 & 45.8 \\
\hline Liana & 2 & 9.1 & 5 & 12.5 & 6 & 10.7 & 7 & 9.7 \\
\hline Total & 22 & 100 & 40 & 100 & 56 & 100 & 72 & 100 \\
\hline
\end{tabular}

Four tree, eight shrubs and single liana species were common to all forest types. Acacia abyssinica, Acacia lahai, Allophyllus abyssinicus, Croton macrostachyus and Teclea nobilis were among the tree species common to all forest types. Maytenus arbutifolia, Carissa edulis, Crateva adansonii, Dodonaea angustifolia, Bersama abyssinica, Rosa abyssinica, Brucea antidysenterica and Vernonia auriculifera were common shrubs in the three forest strata. Petrolobium stellatum was among the liana species that occurred in all forest strata (Table 2, 3 and 4).

Table 2: List of tree species and their existence of similarity in each forest strata

\begin{tabular}{|c|c|c|c|c|c|}
\hline \multirow[b]{2}{*}{ No. } & \multirow[b]{2}{*}{ Scientific name } & \multirow[b]{2}{*}{ Family } & \multicolumn{3}{|c|}{ Occurrence } \\
\hline & & & HDF & MDF & NDF \\
\hline 1 & Acacia abyssinica & Fabaceae & $x$ & $x$ & $x$ \\
\hline 2 & Acacia albida & Fabaceae & - & $x$ & $x$ \\
\hline 3 & Acacia lahai & Fabaceae & $x$ & $\times$ & $x$ \\
\hline 4 & Albizia gummifera & Fabaceae & - & $x$ & $x$ \\
\hline 5 & Allophyllus abyssinicus & Sapindaceae & $x$ & $\times$ & - \\
\hline 6 & Apodytes dimidiate & Icacinaceae & - & $\times$ & - \\
\hline 7 & Bersama abyssinica & Melianthaceae & $\times$ & $\times$ & $\times$ \\
\hline 8 & Cordia africana & Boraginaceae & - & - & $\times$ \\
\hline 9 & Croton macrostachyus & Euphorbiaceae & $\times$ & $\times$ & $\times$ \\
\hline 10 & Dombeya torrid & Sterculiaceae & $x$ & - & $x$ \\
\hline 11 & Dracaena steudner & Dracaenaceae & - & - & $x$ \\
\hline 12 & Ehretia cymosa & Boraginaceae & - & - & $x$ \\
\hline 13 & Ekebergia capensis & Meliaceae & - & - & $\times$ \\
\hline 14 & Erythrina abyssinica & Fabaceae & - & - & $x$ \\
\hline 15 & Eucalyptus camaldulensis* & Myrtaceae & - & - & $\times$ \\
\hline 16 & Euphorbia abyssinica & Euphorbiaceae & - & $\times$ & $\times$ \\
\hline 17 & Euphorbia triucalli & Euphorbiaceae & - & - & $\times$ \\
\hline 18 & Ficus cycomorus & Moraceae & - & $\times$ & $\times$ \\
\hline 19 & Ficus sur & Moraceae & - & $\times$ & $\times$ \\
\hline 20 & Ficus vasta & Moraceae & - & - & $x$ \\
\hline 21 & Juniperus procera* & Cupressaceae & - & - & $\times$ \\
\hline 22 & Maytenus obscura & Celastraceae & - & $\times$ & - \\
\hline 23 & Millettia feruginea & Fabaceae & - & $x$ & $x$ \\
\hline 24 & Morus alba & Moraceae & $x$ & $\times$ & - \\
\hline 25 & Pittosporum viridiflorum & Pittosporaceae & - & - & $\times$ \\
\hline 26 & Podocarpus falcatus & Podocarpaceae & - & - & $\times$ \\
\hline 27 & Prunus africana & Rosaceae & - & - & $x$ \\
\hline 28 & Rhus glutinosa & Anacardiaceae & - & $\times$ & $x$ \\
\hline 29 & Schefflera abyssinica & Araliaceae & - & $x$ & $x$ \\
\hline 30 & Syzygium guinneese & Myrtaceae & - & - & $x$ \\
\hline 31 & Teclea nobilis & Rutaceae & $\times$ & $\times$ & $\times$ \\
\hline Total & & & 8 & 17 & 27 \\
\hline
\end{tabular}

Note: $-=$ Species absent, $x=$ Species present and $*=$ Planted species 
Table 3: List of shrub species and their existence of similarity in each forest strata

\begin{tabular}{|c|c|c|c|c|c|}
\hline \multirow[b]{2}{*}{ No. } & \multirow[b]{2}{*}{ Scientific name } & \multirow[b]{2}{*}{ Family } & \multicolumn{3}{|c|}{ Occurrence } \\
\hline & & & HDF & MDF & $\mathrm{NDF}$ \\
\hline 1 & Acacia brevispica & Fabaceae & - & $x$ & - \\
\hline 2 & Acanthus pubesceae & Acanthaceae & - & - & $\times$ \\
\hline 3 & Acanthus sennii & Acanthaceae & $x$ & - & $x$ \\
\hline 4 & Asparagus flagellaris & Asparagaceae & - & $x$ & - \\
\hline 5 & Brucea antidysenterica & Simaroubaceae & $x$ & $x$ & $\times$ \\
\hline 6 & Calpurnia aurea & Fabaceae & - & $x$ & $x$ \\
\hline 7 & Carissa edulis & Apocynaceae & $\times$ & $x$ & $\times$ \\
\hline 8 & Carissa spinarum & Apocynaceae & - & $x$ & $\times$ \\
\hline 9 & Clausena anisata & Rutaceae & - & $\times$ & - \\
\hline 10 & Clutia abyssinica & Euphorbiaceae & $x$ & - & - \\
\hline 11 & Coffea arabica* & Rubiaceae & - & - & $\times$ \\
\hline 12 & Crateva adansonii & Capparidaceae & $\times$ & $x$ & $x$ \\
\hline 13 & Dodonaea angustifolia & Sapindaceae & $\times$ & $x$ & $\times$ \\
\hline 14 & Dovyalis abyssinica & Flacourtiaceae & - & $x$ & $\times$ \\
\hline 15 & Grewia ferruginea & Tiliaceae & - & $\times$ & $x$ \\
\hline 16 & Hypericum revolutum & Hyperiacaceae & - & $x$ & - \\
\hline 17 & Justica schimperana & Acanthaceae & - & - & $\times$ \\
\hline 18 & Lantana trifolia & Verbenaceae & $x$ & - & - \\
\hline 19 & Maytenus arbitifolia & Celastraceae & $\times$ & $\times$ & $\times$ \\
\hline 20 & Ocimum lamifolium & Lamiaceae & - & $x$ & $x$ \\
\hline 21 & Ocimum urticifolium & Lamiaceae & - & - & $\times$ \\
\hline 22 & Osyris quadripartita & Santalaceae & $\times$ & - & $x$ \\
\hline 23 & Premna schimperi & Lamiaceae & - & - & $x$ \\
\hline 24 & Protea gaguedi & Proteaceae & $\times$ & - & - \\
\hline 25 & Rhamnus prinoides $*$ & Rhamnaceae & - & - & $x$ \\
\hline 26 & Rosa abyssinica & Rosaceae & $x$ & $x$ & $\times$ \\
\hline 27 & Rumex nervosus & Polygonaceae & - & $x$ & $x$ \\
\hline 28 & Sesbania sesban & Fabaceae & - & - & $\times$ \\
\hline 29 & Vernonia amygdalina & Asterraceae & - & $x$ & $\times$ \\
\hline 30 & Vernonia auriculifera & Asteraceae & $\times$ & $x$ & $x$ \\
\hline Total & & & 12 & 18 & 23 \\
\hline
\end{tabular}

Note: $-=$ Species absent, $\times=$ Species present and $*=$ Planted species

Table 4: List of liana species and their existence of similarity in each forest strata

\begin{tabular}{|l|l|l|c|c|c|}
\hline \multirow{2}{*}{ No. } & Scientific name & \multirow{2}{*}{ Family } & \multicolumn{3}{|c|}{ Occurrence } \\
\cline { 3 - 6 } & Capparis tomentosa & Capparidaceae & HDF & MDF & NDF \\
\hline 2 & Clematis longicauda & Ranunculaceae & - & $\times$ & $\times$ \\
\hline 3 & Embelia schimperi & Myrsinaceae & $\times$ & $\times$ & - \\
\hline 4 & Jasminum abyssinicum & Oleaceae & - & $\times$ & $\times$ \\
\hline 5 & Petrolobium stllatum & Fabaceae & $\times$ & $\times$ & $\times$ \\
\hline 6 & Phytolacca dodecandra & Phytolaccaceae & - & - & $\times$ \\
\hline 7 & Rubus steudneri & Rosaceae & - & $\times$ & $\times$ \\
\hline Total & & & 2 & 5 & 6 \\
\hline
\end{tabular}

Note: $-=$ Species absent, $\times=$ Species present 
Table 5: Number of woody species $(\mathrm{N})$ and their corresponding families

\begin{tabular}{|c|c|c|c|c|c|c|c|}
\hline \multirow[b]{2}{*}{ Family } & \multicolumn{2}{|l|}{ HDF } & \multicolumn{2}{|l|}{ MDF } & \multicolumn{2}{|c|}{ LDF } & \multirow[b]{2}{*}{ Total } \\
\hline & $\mathrm{N}$ & $\%$ & $\mathrm{~N}$ & $\%$ & $\mathrm{~N}$ & $\%$ & \\
\hline Acanthaceae & - & - & - & - & 3 & 5.36 & 3 \\
\hline Apocynaceae & - & - & 2 & 5.00 & 2 & 3.57 & 2 \\
\hline Asteraceae & - & - & - & - & 2 & 3.57 & 2 \\
\hline Boraginaceae & - & - & - & - & 2 & 3.57 & 2 \\
\hline Capparidaceae & - & - & 2 & 5.00 & 2 & 3.57 & 2 \\
\hline Celastraceae & - & - & 2 & 5.00 & - & - & 2 \\
\hline Euphorbiaceae & 2 & 9.09 & 2 & 5.00 & 3 & 5.36 & 4 \\
\hline Fabaceae & 3 & 13.64 & 8 & 20 & 9 & 16.07 & 10 \\
\hline Lamiaceae & - & - & - & - & 3 & 5.36 & 3 \\
\hline Moraceae & - & - & 3 & 7.50 & 3 & 5.36 & 4 \\
\hline Myrtaceae & - & - & - & - & 2 & 3.57 & 2 \\
\hline Rosaceae & - & - & 2 & 5.00 & 3 & 5.36 & 3 \\
\hline Rutaceae & - & - & 2 & 5.00 & - & - & 2 \\
\hline Sapindaceae & 2 & 9.09 & 2 & 5.00 & - & - & 2 \\
\hline Others & 15 & 4.55 & 15 & 2.50 & 22 & 1.79 & \\
\hline Total & 22 & 100 & 40 & 100 & 56 & 100 & \\
\hline
\end{tabular}

Note: -Indicates absence of that family

\subsection{Density, Frequency, Dominance and IVI of Woody Species}

The highest relative dominance of trees in the HDF was recorded for Acacia abyssinica (33.16\%) and Morus alba $(20.32 \%)$ with high IVI (88.23\% and 52.43\%) respectively. On the other hand, Allophyllus abyssinicus was the least dominant (0.53\%) tree species that showed an IVI value of 3.5\%. Similarly, Acacia abyssinica and Albizia gummifera were dominant (15.85\% and $10.23 \%$ ) trees with an IVI of $39.62 \%$ and $25.85 \%$ in the MDF respectively. On the other hand, Teclea nobilis was the least dominant tree species in this forest stratum with an IVI of 7.23\%. Euphorbia triucalli and Ficus cycomorus were also the least dominant tree species with the lowest IVI (1.98\% each) in the LDF (Table 6).

The highest relative dominance was recorded for Maytenus arbutifolia $(24.56 \%)$ for shrubs in this forest type. On the other hand, Vernonia auriculifera and Acanthus sennii were recorded lower percent abundance value $(0.29 \%$ and 0.88 ) for shrubs respectively (Table 7). 
Table 6: Relative density, frequency, dominance and IVI of tree species in the three forest strata

\begin{tabular}{|c|c|c|c|c|c|c|c|c|c|c|c|c|}
\hline \multirow[b]{2}{*}{ Species } & \multicolumn{3}{|c|}{ HDF } & \multicolumn{5}{|c|}{ MDF } & \multicolumn{4}{|c|}{ LDF } \\
\hline & $\begin{array}{l}\text { RD } \\
(\%)\end{array}$ & $\begin{array}{l}\text { RF } \\
(\%)\end{array}$ & $\begin{array}{l}\text { RDO } \\
(\%)\end{array}$ & $\begin{array}{l}\text { IVI } \\
(\%)\end{array}$ & $\begin{array}{l}\text { RD } \\
(\%)\end{array}$ & $\begin{array}{l}\text { RF } \\
(\%)\end{array}$ & $\begin{array}{c}\text { RDO } \\
(\%)\end{array}$ & $\begin{array}{l}\text { IVI } \\
(\%)\end{array}$ & $\begin{array}{l}\text { RD } \\
(\%)\end{array}$ & $\begin{array}{l}\text { RF } \\
(\%)\end{array}$ & $\begin{array}{l}\text { RDO } \\
(\%)\end{array}$ & $\begin{array}{l}\text { IVI } \\
(\%)\end{array}$ \\
\hline Acacia abyssinica & 33.12 & 21.95 & 33.16 & 88.23 & 15.85 & 7.92 & 15.85 & 39.62 & 6.18 & 4.79 & 6.18 & 17.15 \\
\hline Acacia albida & - & _ & - & _ & 5.49 & 5.94 & 5.49 & 16.92 & 4.83 & 4.79 & 4.83 & 14.45 \\
\hline Acacia Lahai & 13.35 & 14.63 & 13.37 & 41.35 & 7.62 & 5.94 & 7.62 & 21.18 & 3.28 & 4.19 & 3.28 & 10.75 \\
\hline Albizia gummifera & - & - & - & - & 5.79 & 6.93 & 5.79 & 18.51 & 10.23 & 5.39 & 10.23 & 25.85 \\
\hline Allophylus abyssinicus & 0.53 & 2.44 & 0.53 & 3.5 & 5.18 & 5.94 & 5.18 & 16.3 & - & - & - & - \\
\hline Apodytes dimidiate & _ & - & - & - & 4.88 & 3.96 & 4.88 & 13.72 & - & - & - & - \\
\hline Bersama abyssinica & 10.5 & 8.16 & 13.2 & 31.86 & 2.74 & 4.95 & 2.74 & 10.43 & 0.58 & 1.8 & 0.58 & 2.96 \\
\hline Cordia Africana & _- & - & _ & _ & _ & _- & _- & _- & 3.47 & 3.59 & 3.47 & 10.53 \\
\hline Croton macrostachyus & 9.8 & 11.35 & 7.12 & 28.27 & 9.76 & 7.92 & 9.76 & 27.44 & 5.21 & 4.79 & 5.21 & 15.21 \\
\hline Dombeya torrid & 11.75 & 17.07 & 11.76 & 40.58 & _ & _- & _- & _- & 0.97 & 4.19 & 0.97 & 6.13 \\
\hline Dracaena steudner & - & - & - & - & - & - & - & - & 6.95 & 2.4 & 6.95 & 16.3 \\
\hline Ehretia cymosa & - & - & - & - & - & - & - & - & 2.9 & 1.8 & 2.9 & 7.6 \\
\hline Ekebergia capensis & - & - & - & - & - & - & - & - & 1.54 & 3.59 & 1.54 & 6.67 \\
\hline Erythrina abyssinica & - & - & - & - & - & - & - & - & 1.74 & 3.59 & 1.74 & 7.07 \\
\hline \multicolumn{13}{|l|}{ Eucalyptus } \\
\hline camaldulensis & - & - & - & - & - & - & - & - & 1.74 & 2.4 & 1.74 & 5.88 \\
\hline Euphorbia abyssinica & _ & _ & _ & _ & 3.05 & 2.97 & 3.05 & 9.07 & 3.67 & 4.79 & 3.67 & 12.13 \\
\hline Euphorbia triucalli & - & - & - & - & - & - & - & - & 0.39 & 1.2 & 0.39 & 1.98 \\
\hline Ficus cycomorus & - & - & - & - & 4.57 & 5.94 & 4.57 & 15.08 & 0.39 & 1.2 & 0.39 & 1.98 \\
\hline Ficus sur & - & - & - & - & 3.66 & 5.94 & 3.66 & 13.26 & 1.16 & 4.79 & 1.16 & 7.11 \\
\hline Ficus vasta & - & - & - & - & - & - & - & - & 0.97 & 4.19 & 0.97 & 6.13 \\
\hline Juniperus procera & - & - & - & - & - & - & - & - & 8.11 & 5.99 & 8.11 & 22.21 \\
\hline Maytenus obscura & - & - & - & - & 2.74 & 7.92 & 2.74 & 13.4 & - & - & - & - \\
\hline Millettia ferruginea & _- & _ & _ & _- & 9.15 & 6.93 & 9.15 & 25.23 & 4.05 & 3.59 & 4.05 & 11.69 \\
\hline Morus alba & 17.07 & 12.29 & 23.07 & 52.43 & 6.71 & 6.93 & 6.71 & 20.35 & _ & _ & _ & _ \\
\hline Pittosporum viridiflorum & - & - & - & - & - & - & - & - & 1.93 & 4.79 & 1.93 & 8.65 \\
\hline Podocarpus falcatus & - & - & - & - & - & - & - & - & 2.32 & 1.8 & 2.32 & 6.44 \\
\hline Prunus Africana & - & - & - & - & - & - & - & - & 5.79 & 5.39 & 5.79 & 16.97 \\
\hline Rhus glutinosa & - & - & - & - & 6.71 & 2.97 & 6.71 & 16.39 & 5.79 & 2.4 & 5.79 & 13.98 \\
\hline Schefflera abyssinica & - & - & - & - & 3.96 & 7.92 & 3.96 & 15.84 & 4.83 & 4.19 & 4.83 & 13.85 \\
\hline Syzygium guinneese & - & - & - & - & - & - & - & - & 6.18 & 4.79 & 6.18 & 17.15 \\
\hline Teclea nobilis & 8.55 & 7.32 & 8.56 & 24.43 & 2.13 & 2.97 & 2.13 & 7.23 & 4.83 & 3.59 & 4.83 & 13.25 \\
\hline Total & 100 & 100 & 100 & 300 & 100 & 100 & 100 & 300 & 100 & 100 & 100 & 300 \\
\hline
\end{tabular}

Where: $\mathrm{RD}=$ Relative density, $\mathrm{RF}=$ Relative frequency, $\mathrm{RDO}=$ Relative dominance, $\mathrm{IVI}=$ Important value index 
Table 7: Relative density, frequency, dominance and IVI of shrub species in the three forest strata

\begin{tabular}{|c|c|c|c|c|c|c|c|c|c|c|c|c|}
\hline \multirow[b]{2}{*}{ Species } & \multicolumn{3}{|c|}{ HDF } & \multicolumn{5}{|c|}{ MDF } & \multicolumn{4}{|c|}{ LDF } \\
\hline & $\begin{array}{l}\text { RD } \\
(\%)\end{array}$ & $\begin{array}{l}\text { RF } \\
(\%)\end{array}$ & $\begin{array}{c}\text { RDO } \\
(\%)\end{array}$ & $\begin{array}{l}\text { IVI } \\
(\%)\end{array}$ & $\begin{array}{l}\mathrm{RD} \\
(\%)\end{array}$ & $\begin{array}{l}\text { RF } \\
(\%)\end{array}$ & $\begin{array}{c}\text { RDO } \\
(\%)\end{array}$ & $\begin{array}{l}\text { IVI } \\
(\%)\end{array}$ & $\begin{array}{l}\text { RD } \\
(\%)\end{array}$ & $\begin{array}{l}\text { RF } \\
(\%)\end{array}$ & $\begin{array}{c}\text { RDO } \\
(\%)\end{array}$ & $\begin{array}{l}\text { IVI } \\
(\%)\end{array}$ \\
\hline Acacia brevispica & - & - & - & - & 4.17 & 2.7 & 4.17 & 11.04 & - & - & - & - \\
\hline Acanthus pubescens & - & - & - & - & - & - & - & - & 0.32 & 0.83 & 0.32 & 1.47 \\
\hline Acanthus sennï & 0.88 & 2.74 & 0.88 & 4.5 & - & - & - & - & 0.97 & 5.83 & 0.97 & 7.77 \\
\hline Asparagus flagellaris & - & - & - & - & 4.72 & 4.5 & 4.72 & 13.94 & - & - & - & - \\
\hline \multicolumn{13}{|l|}{ Brucea } \\
\hline antidysenterica & 12.28 & 13.7 & 12.28 & 38.26 & 7.5 & 9.01 & 7.5 & 24.01 & 2.92 & 4.17 & 2.92 & 10.01 \\
\hline Calpurnia aurea & _ & _- & _ & _ & 4.44 & 6.31 & 4.44 & 15.19 & 2.27 & 3.33 & 2.27 & 7.87 \\
\hline Carissa edulis & 12.57 & 10.96 & 12.57 & 36.1 & 25 & 9.01 & 25 & 59.01 & 7.14 & 5.83 & 7.14 & 20.11 \\
\hline Carissa spinarum & - & - & - & - & 3.89 & 5.41 & 3.89 & 13.19 & 0.32 & 0.83 & 0.32 & 1.47 \\
\hline Clausena anisata & - & - & - & - & 4.72 & 4.5 & 4.72 & 13.94 & - & - & - & - \\
\hline Clutia abyssinica & 7.02 & 9.59 & 7.02 & 23.63 & - & - & - & - & - & - & - & - \\
\hline Coffea Arabica & - & - & - & - & - & - & - & - & 1.62 & 5 & 1.62 & 8.24 \\
\hline Crateva adansonï & 7.89 & 8.22 & 7.89 & 24 & 4.17 & 6.31 & 4.17 & 14.65 & 5.19 & 5.83 & 5.19 & 16.21 \\
\hline \multicolumn{13}{|l|}{ Dodonaea } \\
\hline angustifolia & 7.31 & 10.96 & 7.31 & 25.58 & 8.06 & 12.62 & 8.06 & 28.74 & 6.17 & 5.83 & 6.17 & 18.17 \\
\hline Dovyalis abyssinica & - & - & - & - & 5 & 3.6 & 5 & 13.6 & 3.25 & 3.33 & 3.25 & 9.83 \\
\hline Grewia ferruginea & - & - & - & - & 5.28 & 3.6 & 5.28 & 14.16 & 2.6 & 5.83 & 2.6 & 11.03 \\
\hline Hypericum revolutum & - & - & - & - & 4.72 & 7.21 & 4.72 & 16.65 & - & - & - & - \\
\hline Justica schimperana & - & - & - & - & - & - & - & - & 7.14 & 2.5 & 7.14 & 16.78 \\
\hline Lantana trifolia & 5.56 & 8.22 & 5.56 & 19.34 & - & - & - & - & - & - & - & - \\
\hline Maytenus arbutifolia & 24.56 & 13.7 & 24.56 & 62.82 & 8.06 & 6.31 & 8.06 & 22.43 & 9.42 & 5 & 9.42 & 23.84 \\
\hline Ocimum Lamifolium & - & - & - & - & 3.61 & 4.5 & 3.61 & 11.72 & 2.27 & 3.33 & 2.27 & 7.87 \\
\hline Ocimum urticifolium & - & - & - & - & - & - & - & - & 1.62 & 6.67 & 1.62 & 9.91 \\
\hline Osyris quadripartita & 10.82 & 6.85 & 10.82 & 28.49 & - & - & - & - & 7.47 & 2.5 & 7.47 & 17.44 \\
\hline Premna schimperi & - & - & - & - & - & - & - & - & 8.12 & 5.83 & 8.12 & 22.07 \\
\hline Protea gaguedi & 4.39 & 6.85 & 4.39 & 15.63 & - & - & - & - & - & - & - & - \\
\hline Rhamnus prinoides & - & - & - & - & - & - & - & - & 4.22 & 3.33 & 4.22 & 11.77 \\
\hline Rosa abyssinica & 6.43 & 6.85 & 6.43 & 19.71 & 3.06 & 5.41 & 3.06 & 11.53 & 2.6 & 4.17 & 2.6 & 9.37 \\
\hline Rumex nervosus & - & - & - & - & 2.22 & 3.6 & 2.22 & 8.04 & 9.09 & 5 & 9.09 & 23.18 \\
\hline Sesbania sesban & - & - & - & - & - & - & - & - & 7.14 & 5.83 & 7.14 & 20.11 \\
\hline Vernonia amygdalina & - & - & - & - & 1.39 & 5.41 & 1.39 & 8.19 & 3.25 & 4.17 & 3.25 & 10.67 \\
\hline Vernonia auriculifera & 0.29 & 1.37 & 0.29 & 1.95 & - & - & - & - & 4.87 & 5 & 4.87 & 14.74 \\
\hline Total & 100 & 100 & 100 & 300 & 100 & 100 & 100 & 300 & 100 & 100 & 100 & 300 \\
\hline
\end{tabular}

Where: $\mathrm{RD}=$ Relative density, $\mathrm{RF}=$ Relative frequency, $\mathrm{RDO}=$ Relative dominance, IVI =Important value index

\subsection{Woody Species Diversity, Richness and Evenness}

Richness of woody species decreased from the low disturbed forest (56) to the MDF and HDF (40 and 22) species respectively. The Shannon Wiener diversity index for LDF was higher (3.76) followed by MDF and LDF (3.49 and 2.84) respectively. Evenness (Shannon equitability) values of the three forest types were $0.93,0.95$ and 0.92 for LDF, MDF and HDF respectively (Table 8). As far as the Shannon Wiener diversity index of woody species by their growth habit is concerned, tree diversity in the LDF was significantly higher (0.30) than HDF and MDF strata (d.f. $=2 ; p \leq 0.05$ ). With regard to shrubs, the HDF is higher in diversity $(0.18)$ than MDF and LDF, but they are not significant (d.f. $=2 ; \mathrm{p} \geq 0.05$ ) whereas liana diversity between the MDF $(0.32)$ and LDF $(0.30)$ were not vary significantly (d.f. $=2 ; \mathrm{p} \leq 0.05$ ). Liana diversity is lower in HDF $(0.25)$ than MDF and LDF patches (Table 9). 
Table 8: Summary of woody species richness, diversity and evenness in three forest strata

\begin{tabular}{lccc}
\hline & \multicolumn{3}{c}{ Forest strata } \\
\cline { 2 - 4 } Diversity indices & LDF & MDF & HDF \\
\hline Woody species richness (S) & 56 & 40 & 22.84 \\
Shannon Weiner diversity index (H') & 3.76 & 3.49 & 3.09 \\
Maximum diversity (H max) & 4.03 & 3.69 & 0.92 \\
Shannon evenness (J) & 0.93 & 0.95 & \\
\hline
\end{tabular}

Table 9: Mean ( \pm Std.) of Shannon Weiner diversity index of trees, shrubs and lianas

\begin{tabular}{lccc}
\hline & \multicolumn{3}{c}{ Forest strata } \\
\cline { 2 - 4 } Growth habit & HDF & MDF & LDF \\
\hline Tree & $0.16^{\mathrm{b}} \pm 0.05$ & $0.24^{\mathrm{a}} \mathrm{b} \pm 0.11$ & $0.30^{\mathrm{a}} \pm 0.06$ \\
Shrub & $0.18^{\mathrm{a}} \pm 0.08$ & $0.15^{\mathrm{a}} \pm 0.06$ & $0.13^{\mathrm{a}} \pm 0.06$ \\
Liana & $0.25^{\mathrm{b}} \pm 0.06$ & $0.32^{\mathrm{a}} \pm 0.12$ & $0.30^{\mathrm{a}} \pm 0.08$
\end{tabular}

Note: Different letters across rows show significance difference between forest types $(\mathrm{p} \leq 0.05)$

\subsection{Diameter Distribution of Trees and Shrubs}

The diameter distribution of trees in all the forest strata followed the normal inverted $\mathrm{J}$ curve. The density per hectare of trees in various diameter classes decreased with increasing diameters. The highest densities were belongs to the lower diameter classes $(10-15 \mathrm{~cm}, 15.01-20 \mathrm{~cm}$ and $20.01-25 \mathrm{~cm})$ for trees in three forest strata. Similar trend was also observed for shrubs that shrub density per hectare was high in lower diameter classes. Tree density per hectare for all forest strata continues increasing up to the last diameter class $(\geq 50.01 \mathrm{~cm})$. However, in the HDF it continues up to $30.01-35 \mathrm{~cm}$ in diameter (Figure 2 and 3 )

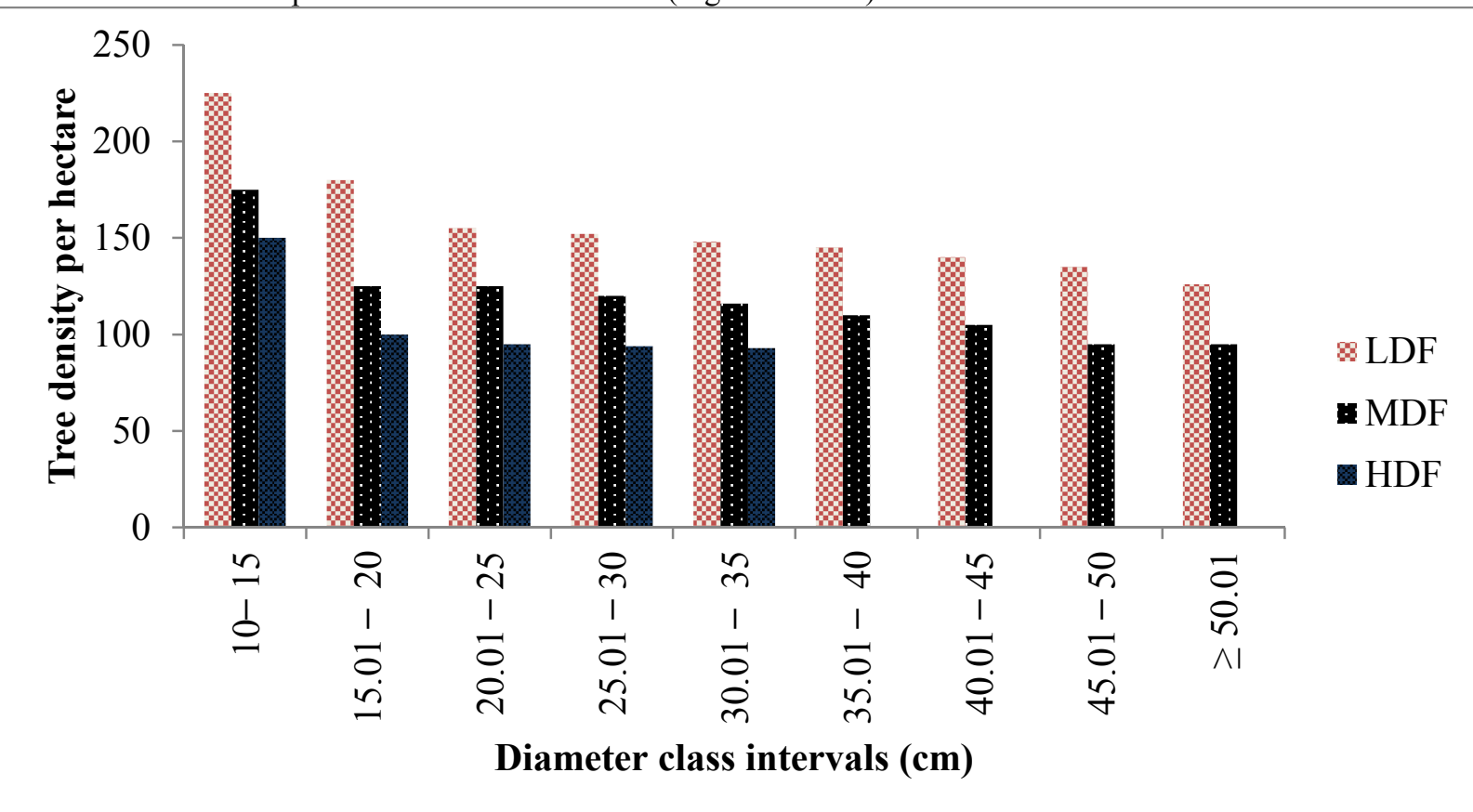

Figure 2: Diameter distribution of trees $(\geq 10 \mathrm{~cm})$ in three forest strata 


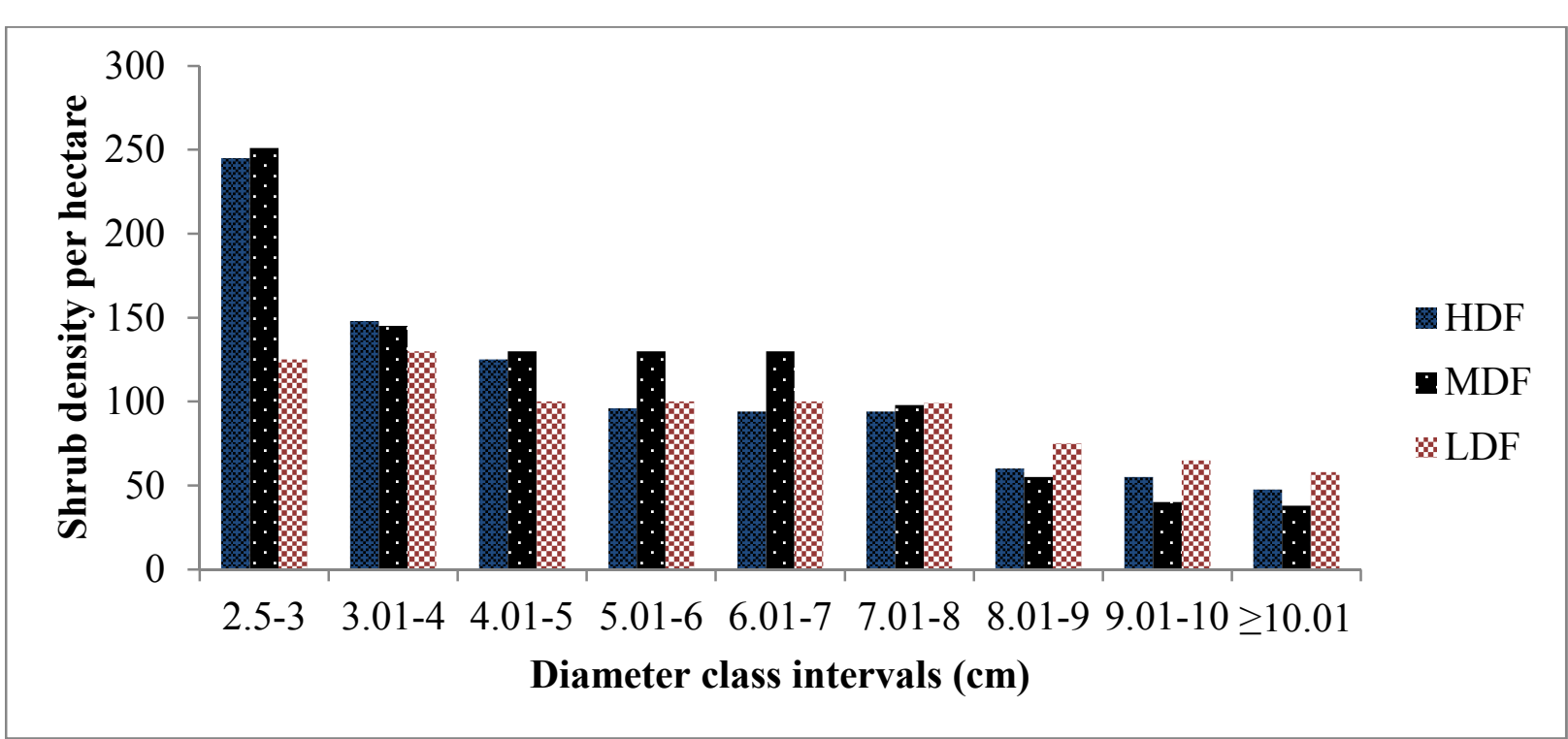

Figure 3: Diameter distribution of shrubs $(\geq 2.5 \mathrm{~cm})$ in three forest strata

There was a significant difference in tree density between HDF and LDF (d.f. $=2 ; \mathrm{p} \leq 0.05$ ). However, there was no significance difference between LDF and MDF. Similarly, there were no significance differences between MDF and HDF (Table 10).

Table 10: Mean density per hectare $( \pm$ Std. $)$ for trees, shrubs and lianas in three forest strata

\begin{tabular}{lcccr}
\hline & \multicolumn{3}{c}{ Forest strata } \\
\cline { 2 - 5 } Growth habit & MDF & HDF & p - value \\
\hline Tree & LDF & $44.88 \pm 30.98$ & $33.57 \pm 18.75$ & 0.004 \\
Shrub & $81.13 \pm 49.21$ & $50.33 \pm 46.51$ & $66.08 \pm 51.89$ & 0.063 \\
Liana & $33.48 \pm 21.88$ & $43.60 \pm 22.63$ & $9.00 \pm 1.41$ & 0.054 \\
\hline
\end{tabular}

Table 11: Average height $(\mathrm{m})$ of woody species for canopy layer class in three forest strata

\begin{tabular}{lccccc}
\hline & \multicolumn{5}{c}{ Forest strata } \\
\cline { 2 - 5 } Canopy layer class & HDF & MDF & NDF & Over all & Percentage $(\%)$ \\
\hline Low $(<5 \mathrm{~m})$ & 56 & 15 & 22 & 93 & 24.41 \\
Medium $(5-15 \mathrm{~m})$ & 43 & 65 & 49 & 157 & 41.21 \\
Higher $(\geq 15 \mathrm{~m})$ & 9 & 32 & 90 & 131 & 34.38 \\
\hline Total & \multicolumn{7}{c}{381} & 100 \\
\hline
\end{tabular}

\section{DISCUSSION}

Human-induced disturbances are the major causes for changes in forest structure and composition (Kumar \& Ram 2005), and the extents of these effects are dependent on the type and severities of the disturbances (Chown, 2010). In this study some trees, shrubs and lianas are common to the forest patches. The presence of such common species may be due to similar ecological habitat of those woody species and the close distance $(50 \mathrm{~m})$ between sampled plots. The present result indicates that, shrub and tree species were found to be higher than other plant life forms in the study area. Generally, shrubs constituted the major life form of woody species in the MDF and HDF as compared to trees and lianas. On the other hand, trees constituted the major life form in the LDF. The existence of differences between the forest patches which in turn implies the importance in terms of floristic diversity and needs attention from a conservation point of view. As reported by (Dereje, 2007) anthropogenic forest disturbance and other environmental factors such as, soil physical and chemical properties and others have sound effects on patterns of natural forest vegetation.

With regards to families of woody species in the study area, Fabaceae was the most common in all forest patches (Table 5). Similar study by (Alemayehu, 2002) at Tara Gedam forest indicated the dominance of Fabaceae family. The authors suggest that, this might be attributed due to the adaptation potential of Fabaceae families' to the area.

From the result section, some tree and shrub species like Allophyllus abyssinicus, Teclea nobilis, Euphorbia triucalli, Ficus cycomorus were the least dominant tree species in the LDF and Vernonia auriculifera and Acanthus sennii were the least dominant shrub species in this forest patch. For those of all tree and shrub species listed in Table 6 and 7 with lower IVI needs priority for conservation to enhance their normal distribution in the forest. 
The species with the least occurrence like Vernonia auriculifera, and Allophyllus abyssinicus in the HDF, Acanthus pubescens and Carissa spinarum in the LDF were recorded in some plots. Species of such least frequency need to be given priority in conservation to enhance their frequency distribution. The most frequently observed species along plots of the highly disturbed forest were exhibit the shrub life form. The high frequency indicates even distribution of the species in the forests and variation in frequency between species may be attributed to habitat differences, habitat preferences among the species, species characteristics for adaptation, degree of exploitation and conditions for regeneration (Demel, 1995).

As expected, the present study demonstrated that woody species diversity vary along disturbance gradients in the studied forests. In all forest types species richness decreased from the LDF through the MDF to the HDF. The HDF is the least in species evenness and richness (Table 8). The authors suggest that, high evenness value in all forest strata from the result may be due to similar adaptation potential of woody species in that particular ecosystem. From each forest strata inventoried, the LDF (church forest) was found to be relatively high in species richness and diversity. The results of the present study agrees with (Tamirat,1993) who stated that, church forests possess diverse woody species and contain good indigenous plant wood stock as compared to biggest continuous forests in the highlands of Ethiopia. It is often observed that increased fragmentation of natural habitats by human disturbances leads to reduced species richness, and that many variables cause species loss along that gradient (Mc Kinney, 2002).

Low species diversity and evenness for HDF from the result is due to excessive anthropogenic disturbances that inhibit plant growth and excessive exploitation of woody species. Similarly, Desalegn \& Zerihun (2005) stated that low species diversity and evenness could be attributed to the presence of forest disturbance and absence of adequate nutrient and moisture. Generally, it is possible to conclude that difference in woody species richness, diversity and evenness between forest types is basically due to the variation in the intensity of anthropogenic forest disturbance as a main factor.

The difference in woody species diversity in different growth habits may be due to the influence of human disturbances in the forests (Table 9). In the case of trees, the occurrence of significant differences in diversity between the three forest types clearly demonstrates the level of human disturbance on different plant life forms. Comparing this result to other related works, Muhanguzi et al. (2007) documented similar result that a decline trend in tree species diversity from the LDF to the MDF to HDF. The current study also confirms the work of other authors that trees constitute the predominant woody life forms in natural forests that has not under gone different forms of human modifications (Anning et al., 2008).

The diameter distribution of trees and shrubs in all the forest strata followed the normal inverted $\mathrm{J}$ curve. The normal inverted $\mathrm{J}$ shape pattern of trees and shrubs in all forest type may be due to shade effect on small regenerates and also diameter and density are not directly proportional to each other. Similar result was explained by (Nord \& Cao, 2006) stated that, deforestation and tree shade effect causes the decrease in the density of woody species in a natural forest. Tree species which had included under higher diameter class i.e. $\geq 50.01 \mathrm{~cm}$ mainly includes species of Prunus africana, Ficus vasta, Albizia gummifera and Junipures procera which were the tallest tree species that dominantly found within the low disturbed forest (Gumdri Abo church forest) and form the upper canopy of this forest strata.

There was a significant difference in tree density between HDF and LDF (d.f. $=2 ; \mathrm{p} \leq 0.05$ ). From this result it is clear that the HDF has lower tree density and needs an immediate conservation action. Generally, there was a decreasing trend in densities of woody species with increasing disturbance i.e. from the LDF through the MDF to the HDF. According to Muhanguzi et al., 2007, the result of this work realizes that, areas that support many species also support many individuals.

The vertical structure of woody species in the three forest strata was not uniform i.e. varied from one forest type to another mainly due to the influence of anthropogenic disturbance. The effect of disturbance on height class was clearly evident in the study area that as human induced forest disturbance increases, the height of trees and shrubs highly decreasing. This pattern is supported by the work of (Pereira et al., 2001) that states the direct removal of trees by logging and farming activities were directly responsible for the decrease in height in disturbed forests. From the result it is possible to conclude that most species were attained the medium canopy layer class $(5-15)$ in the three forest types.

\section{Conclusion}

In Gumdri Abo Natural Forest (GANF), a total of 73 woody plant species, representing 40 families and 62 genera were recorded. Among recorded families Fabaceae was found dominant in species composition in the three forest types/stratum. In terms of plant growth habit, majority of woody species were shrubs $(45.8 \%)$ followed by trees (44.4\%). Diversity and richness of woody species in all life forms decreased with increasing anthropogenic forest disturbance. Diameter distribution of woody species followed the normal inverted J shape curve in all forest types. The result of the study revealed that human disturbance has negative influence on species richness, diversity and structure of moderately and heavily disturbed forests. Regarding to vegetation inventory, woody species richness, 
diversity and all structural parameters decreased form low disturbed forest to heavily disturbed forest. This is an indicator of species distribution pattern along human disturbance that significantly alter woody species composition in disturbed forests particularly in the heavily disturbed forest. Therefore, forest conservation programs should be undertaken in those disturbed forests as similar as the low disturbed forest type.

\section{ACKNOWLEDGEMENTS}

Authors are grateful to the management and staff members of Wondo Genet College of Forestry and Natural Resources (WGCF-NR) for their support during the study. The logistical support provided by the Hawssa University WGCF-NR which is financed by Ethiopian ministry of finance and economic development is gratefully acknowledged. Authors would like to also thank Mr. Haileyesus Gelaw and Mr. Adamu Worku for their support in the field data collection from Hawassa University WGCF-NR.

\section{References}

Abed, T. \& Stephens, N.C., 2003. Tree measurement manual for farm foresters. M. Parsons ( $2^{\text {nd }}$ edn.), National Forest Inventory, Bureau of Rural Sciences, Canberra.

Addo Fordjour, P. Obeng, S. Anning, A. K. \& Addo, M. G., 2009. Floristic composition, structure and natural regeneration in a moist semi-deciduous forest following anthropogenic disturbances and plant invasion. International Journal of Biodiversity and Conservasion, 2: 21 - 37.

Alemayehu, W., 2002. Opportunities, constraints and prospects of the Ethiopian Orthodox Tewahido Churches in conserving forest resources: the case of churches in South Gondar, Northern Ethiopia. M.Sc. Thesis, Swedish University of Agricultural Sciences.

Chown, S.L. 2010.Temporal biodiversity change in transformed landscapes: a southern African perspective. Philosophical Transactions of the Royal Society, Biological Sciences, 365: 3729-3742.

Anning, A.K., Akyeampong, S., Addo-Fordjour, P., Anti, K.K., Kwarteng, A. and Tettey, Y.F., 2008. Floristic composition and vegetation structure of the KNUST Botanic Garden, Kumasi, Ghana. Journal of Science and Technology (Ghana), 28(3), pp.103-122.

Armesto, J. J. and Pickett, S. T. A., 1985. Experiments on disturbance in old-field plant communities: impact on species richness and abundance. Ecology, 66: 230-240.

Bekele, A., 2007. Useful trees and shrubs of Ethiopia: Identification, Propagation and Management for 17 Agroclimatic Zones. Technical Manual No 6. RELMA, Nairobi, Kenya. 552 pp.

Coetzee JA. Phyto geographical Aspects of the Montane Forests of the Chain of Mountains on the Eastern Side of Africa. Erdwissenschaftliche Forschung. 1978; 11: 482-494p.

Dangila Woreda Agricultural Office (DWAO), 2014. Awi zone, Annual Report for the year 2013/2014.

Demel, T., 1995. Floristic composition of Wof-Washa natural forest, Central Ethiopia: Implication for the conservation of biodiversity. Report, 106, 127-147.

Dereje, D., 2007. Floristic Composition and Ecological Study of Bibita Forest (Gura Farda), Southwest Ethiopia , M.Sc. Thesis, Addis Ababa University, Addis Ababa.

Ethiopian Forestry Action program (EFAP), 1994.). The Challenge for Development, Summary of Final report (Vol. 3). Ministry of Natural Resources, Addis Ababa, Ethiopia.

Ensermu, K., \& Teshome, S., 2008. Interface of Regeneration, structure, Diversity and use of some plant species in Bonga Forest: a Reservoir for wild coffee gene pool. Ethiopian Journal of forestry Sciences., 31(2), 121134.

Food and Agriculture Organization (FAO), 2015. Global Forest Resources Assessment and Current status of forest resources report of Ethiopia.

Feyera, S., \& Demel, T., 2003. Diversity, community types and population structure of woody plants in Kimphee Forest, a virgin nature reserve in Southern Ethiopia. Ethiopian Journal of Biological Sciences 2(2): 169-187.

Hegde, R. \& Enters, T., 2000. Forest products and household economy: a case study from Mudumalai Wildlife Sanctuary, Southern India. Environmental Conservation, 27: 250- 259.

Huston, M. A., 1994. Biological diversity. The Existence of Species on Changing Landscapes. Cambridge University Press, Cambridge, 681Pp.

Kent, M. \& Coker, P., 1992. A practical approach for Vegetation Description and Analysis, John Wiley and Sons, New York.

Kumar, A. \& Ram, J. 2005. Anthropogenic disturbances and plant biodiversity in forests of Uttaranchal, central Himalaya. Biodiversity and Conservation, 14: 309-331.

Magurran, A.E. , 1988. Ecological Diversity and its Measurement. Chapman and Hall. London.

McKinney ML. 2002. Urbanization, biodiversity, and conservation. BioScience. 52(10):883-890.

Mueller-Dombois, D. \& Ellenberg, H., 1974. Aims and Methods of Vegetation Ecology.John Wiley \& Sons, Inc., New York. pp 547.

Muhanguzi, J. O., Hosea, D. R., Oryem-Origa, H., 2007. The effect of human disturbance on tree species 
composition and demographic structure in Kalinzu 107 (81-95).

Nord-Larsen, T. \& Cao, Q. V., 2006. A diameter distribution model for even-aged beech in Denmark. Forest Ecology and Management, 231: pp 218-225.

Palit D, Pal S \& Chanda S., 2012. Diversity and richness of plants in Darjeeling Himalaya with an eye on Gaddikhana forest beat, Senchal east zone forest range, Darjeeling. Indian Journal of forestry 35: pp 39-44.

Pereira, M. C. A., Araujo, D. S. D. \& Pereira, O. J., 2001. Structure of a scrub community of restinga of Barra de Marica - RJ, Rio de Janeiro - Brazil. Revta Brasil. Botany, 24(3): 237- 281.

Ram J, Kumar A \& Bhatt J., 2004. Plant diversity in six forest types of Uttaranchal, Central Himalaya, India. Current Science. 86: pp 975-978.

Ramirez-Marcial, N., Gonzalez-Espinosa, M., Williams-Linera, G., 2001. Anthropogenic disturbance and tree density in Montane Rain Forests in Chiapas, Mexico. Ecology and Management. 154: pp 311-32.

Richards, P.W., 1996. Africa, the 'Odd man out'. Meggers, B.J., Ayensu, E.S. \& Duckworth, D. (eds.), Tropical forest ecosystems in Africa and South America, a comparative review. Smithsonian Inst. Press, Washington DC.

Sang, W., 2009. Plant diversity patterns and their relationships with soil and climatic factors along an altitudinal gradient in the middle Tianshan Mountain area, Xinjiang, China. Ecological Resources, 24: pp 303-314.

Sebsebe D., 1997. Flora of Ethiopia and Eritrea volume 6 The National Herbarium, Biology Department, Science Faculty, Addis Ababa University, Ethiopia, and Department of Systematic Botany, Uppsala University, Sweden.

Sebsebe, D. \& Friis, I., 2009. Natural vegetation of the Flora area: Flora of Ethiopia and Eritrea. (Hedberg, I., Friis, I. \& Persson, E., eds), pp. 28-29, the National Herbarium, Addis Ababa University, Addis Ababa.

Snowdon, P., Raison, J., Keith, H., Ritson, P., Grierson, P., Adams, M., Montagu, K., Bi HQ.

Burrows, W. \& Eamus, D., 2002. Protocol for sampling tree and stand biomass. National Carbon accounting System, Technical report no. 31. Canberra: Australian Greenhouse Office.

Tamirat, B., 1993. Studies on Remenant Afromontane forests on the central plateau of Shewa, Ethiopia. Doctoral Dissertation Uppsala University.Upsala,Sweden.

Vivero. J. L., Ensermu, K. \& Sebsebe, D., 2006. Progress on the Red list of plants of Ethiopia and Eritrea: Conservation and biogeography of endemic flowering taxa: Taxonomy and Ecology of African Plants, their Conservation and Sustainable. Proceedings of the 17 $7^{\text {th }}$ Aetfat congress, Addis Ababa, Ethiopia. Pp 761-778.

Wana, D. \& Woldu, Z., 2005. Vegetation of Chencha highlands in southern Ethiopia. Ethiopian Journal of Science, 28(2), pp.109-118. 